\title{
Comparison of Collision Detection Techniques for High-Speed Industrial Robot Actuators with Overload Clutch
}

\author{
Frederik Ostyn \\ Electromech., Systems and Metal Eng. \\ Ghent University \\ Core Lab EEDT-DC, Flanders Make \\ Ghent, Belgium \\ frederik.ostyn@ugent.be
}

\author{
Bram Vanderborght \\ Robotics and Multibody Mechanics \\ Vrije Universiteit Brussel \\ Core Lab R\&MM, Flanders Make \\ Brussels, Belgium \\ bram.vanderborght@vub.be
}

\author{
Guillaume Crevecoeur \\ Electromech., Systems and Metal Eng. \\ Ghent University \\ Core Lab EEDT-DC, Flanders Make \\ Ghent, Belgium \\ guillaume.crevecoeur@ugent.be
}

\begin{abstract}
High-speed industrial robots can cause severe damage in the event of a collision. In order to deploy them in unstructured environments, they require overload protection and sensitive collision detection. A qualitative and quantitative comparison of different detection techniques is presented based on high-speed collision experiments. A high-speed industrial robot actuator with overload clutch was instrumented with various sensors for this purpose. Limit switches and secondary encoders required the clutch to decouple prior to detection. Proprioceptive techniques were successfully implemented but showed lower sensitivity compared to accelerometer based collision detection.

Index Terms-collision detection, actuator, robot, clutch
\end{abstract}

\section{INTRODUCTION}

Robots have the risk to collide, especially while operating in unknown or unstructured environments. Safety is the prior concern when humans interact with collaborative robots. Speeds are kept low according to safety standards such as ISO/TS 15066:2016. On the other hand, high-speed industrial robots, which for safety reasons have to operate in cages, still have the risk to create (internal) damage when colliding. The Combined Friction Cam Clutch (CFCC) was introduced in [1] as a means to increase mechanical compliance upon impact without compromising the dynamic performance in nominal operation. The clutch has sufficient residual torque to counteract gravity once decoupled and allows an automatic reset. Generally speaking, clutches allow a significant but finite relative angle difference between input and output side upon collision. While they protect the drive train from overload, the decoupling of the clutch compromises the kinematic configuration of the robot. The collision must therefore be detected in order for the robot to react accordingly, for instance by initiating an automatic reset as discussed in [1].

Collision detection techniques rely on different sensor architectures and use either model-based or data-driven techniques to identify collisions. Collision detection strategies relying on motor position and current alone are often referred to as 'sensorless' or proprioceptive solutions as both are usually already

This work was supported by the BOF project 01N02716. monitored during nominal robot control [2]. In addition, data of joint and/or wrist force/torque sensors, accelerometers and gyroscopes can improve the detection speed and accuracy (avoiding false positives and undetected collisions) but add to the cost and complexity of the detection solution. Torque sensors measure the load torque directly. They rely on strain gauges or capacitance monitoring as a means to measure torque induced deformations in a base structure. These devices are sophisticated and come at a cost. Reducing the cost as well as their size and complexity is ongoing research [3]. In comparison, MEMS accelerometers and gyroscopes are cheaper and easier to install as they come as system on chip and are mass produced. Although they do not measure torque directly, they can be used to detect collisions [4]. The challenge with these devices is in their calibration. Combinations of force/torque sensors with accelerometers are also used [5]. Limit switches and (low resolution) secondary encoders are an option as well if the actuator is equipped with an overload clutch. Limit switches monitor if the input and output flange of the clutch rotate with respect to each other. The signal is either one or zero and the interpretation straightforward. Secondary encoders serve a similar purpose. They output the actual angular difference between the input and output clutch flange. They are more complex than limit switches but offer higher accuracy.

Data-driven techniques do not require a model of the robot. They rely on filters and dynamic thresholds to detect a collision. Examples are the work by Geravand et al. [6] and Indri et al. [7]. Sharkawy et al. used neural networks (NN) to identify collisions based on motor encoder data [8]. In order to train the model, the robot must (temporally) be instrumented with additional direct force/torque sensors. Model-based techniques require dynamic models describing the state of the robot and residual or disturbance observers to estimate this state and other collision relevant parameters [9]. As summarized in [2], the most common observers are energy, velocity or momentum based. Since its introduction by De Luca et al. in [10], the momentum observer has been extended to distinguish between 
intended contact and unexpected collision [11]. Furthermore, it can also be used relying on motor currents instead of direct joint torque sensing by including a non-linear friction model of the drivetrain [12]. Both extensions were combined in [13]. A second order sliding mode extension was proposed by Garofalo et al. [14]. Birjandi et al. suggested to use Kalman filtering to estimate load acceleration [15], [16]. A simplified integrator model was introduced with motor encoder and load accelerometer data as input. The full dynamic model is subsequently used to calculate the external load directly. The main advantages of this method are the avoidance of delays introduced by the momentum observer and the preprocessing of its input, often obtained by low pass filtering differentiated position encoder data.

The contribution of this article is in the evaluation of different sensor architectures and the appropriate data-driven or model-based techniques to detect collisions at high speed $(300 \% \mathrm{~s})$. The aim is to have a low cost solution, excluding the use of joint torque sensors. How this affects the modelbased techniques will be discussed in detail. Methods requiring training (such as [8]) are also excluded in this study. An actuator with a Combined Friction Cam Clutch (CFCC) as introduced in [1] serves as use case for this study. The results are however not necessarily restricted to this type of clutch.

This article is organised as follows: first, the different collision detection techniques are introduced in Section II The actuator with overload clutch that is used as experimental platform is presented next (Section III-A). Experimental results including a qualitative and quantitative comparison of the different collision detection techniques are documented in Section ПII-B-D. The article is concluded in Section IV

\section{COLLISION DETECTION TECHNIQUES}

Several sensor architectures and detection techniques are presented next. Their parameters are listed in Table I

\section{A. Load cell}

An external torque sensor measures the external collision torque directly. A force sensor that is pressed by a known load arm with length $L_{\mathrm{LC}}$ can serve a similar purpose. An external device is not considered as viable option to be used in an actual robot for collision detection purposes. The closest alternative is a joint or wrist force/torque sensor. The load cell is used in this study as benchmark to evaluate the sensor architectures to follow.

\section{B. Proximity switch or low resolution encoder}

A proximity switch measures if material is present in front of it or not. Such a device is often used as limit switch. In this application, it detects if the clutch input and output side rotate with respect to each other. Proximity switches are low cost and their interpretation is straightforward: the output is either one or zero. This instrumentation comes with the following disadvantages (1) only collisions resulting in clutch disengagement are detected and (2) the relative angle difference that triggers a detection depends on the accuracy with which they were installed.

The separation of the clutch halves can also be monitored with an encoder. Even low resolution (and hence lower cost) encoders will outperform a limit switch. They however need more computational power. Only collisions resulting in clutch disengagement are detected.

\section{Data-driven collision detection based on motor currents or accelerometer data}

Geravand et al. suggest the use of a high-pass filter to identify unintentional collisions while monitoring the motor torque $\tau_{m}$ or motor current $i_{m}$ [6]. In the following, a high-pass Butterworth filter of third order is used. Collisions resulting in $>15 \mathrm{~Hz}$ responses can be detected:

$$
\tau_{m, \mathrm{HPF}}\left(t_{k}\right)=\frac{\sum_{l=0}^{3} b_{\mathrm{HPF}, l} \tau_{m}\left(t_{k}-l \Delta t\right)}{1+\sum_{l=1}^{3} a_{\mathrm{HPF}, l} \tau_{m, \mathrm{HPF}}\left(t_{k}-l \Delta t\right)}
$$

with $\Delta t$ the sampling time and $a_{\mathrm{HPF}, l}, b_{\mathrm{HPF}, l}$ depending on the filter frequency. Their values are listed in Table I. Note that the filter has a sampling time of $1 \mathrm{~ms}$ versus the $12 \mathrm{~ms}$ sample time reported in [6].

A collision is detected if the filtered motor torques $\left|\tau_{m, \mathrm{HPF}}\right|$ exceed a dynamic detection threshold

$$
\tau_{m, \mathrm{HPF}, \mathrm{TH}}=\tau_{\mathrm{HPF}, 0}+\tau_{\mathrm{HPF}, 1} \frac{\left|\dot{q}_{m, d}\right|}{v_{\max }}+\tau_{\mathrm{HPF}, 2} \frac{\left|\ddot{q}_{m, d}\right|}{a_{\max }}
$$

with $\tau_{\mathrm{HPF}, 0}$ chosen to cover static conditions, $\dot{q}_{m, d}$ and $\ddot{q}_{m, d}$ the commanded motor velocity and acceleration respectively, $v_{\max }$ and $a_{\max }$ the maximum motor velocity and acceleration and $\tau_{\mathrm{HPF}, 1}$ and $\tau_{\mathrm{HPF}, 2}$ tuning parameters. In order to simplify the comparison of different techniques, only the static condition is used $\left(\tau_{\mathrm{HPF}, 1-2}=0\right)$. The threshold is tuned as sensitive to collisions as possible while avoiding false positives during nominal movement.

Load accelerometer data can be used alternatively. The same $15 \mathrm{~Hz}$ Butterworth high-pass filter can be used to eliminate low frequency signal content such as gravitational acceleration. A similarly constructed dynamic threshold as Eq. (2) can be used. As stated before, only the static condition will be used.

\section{D. 'Sensorless' model-based collision detection using a mo- mentum observer}

Model-based techniques use dynamic models describing the state of the robot and observers to estimate this state. The dynamics of the complete actuator can be described based on a set of Lagrange-Euler equations with all parameters reduced to the load side:

$$
\begin{aligned}
J_{l} \ddot{q}_{l}+D_{l} \dot{q}_{l}+g\left(q_{l}\right) & =\tau_{\mathrm{ext}}+\tau_{c} \\
J_{m} \ddot{q}_{m}+\tau_{f}^{\prime} & =\tau_{m}-\tau_{c}
\end{aligned}
$$

with $J_{l}$ and $J_{m}$ being the clutch output and load and the motor inertia respectively. $q_{l}$ and $q_{m}$ are the accompanying rotary positions, $D_{l}$ a viscous friction coefficient (mainly due to the friction in the cross roller bearing), $\tau_{\mathrm{ext}}$ is the external torque, $\tau_{c}$ the clutch torque (derived in [1]), $g\left(q_{l}\right)$ gravitational torque 
TABLE I

COLLISION DETECTION AND CLUTCH PARAMETERS. ALL QUANTITIES ARE EXPRESSED WITH RESPECT/REDUCED TO THE LOAD SIDE.

\begin{tabular}{|l|l|l|}
\hline Parameter & Symbol & Value \\
\hline Sample time [ms] & $\Delta t$ & 1 \\
Maximal velocity [\%/s] & $v_{\max }$ & 300 \\
Maximal acceleration [rad/s ${ }^{2}$ ] & $a_{\max }$ & 60 \\
Filter coefficient & $b_{\mathrm{HPF}, 0}$ & 0.910 \\
Filter coefficient & $b_{\mathrm{HPF}, l}$ & -2.730 \\
Filter coefficient & $b_{\mathrm{HPF}, 2}$ & 2.730 \\
Filter coefficient & $b_{\mathrm{HPF}, 3}$ & -0.910 \\
Filter coefficient & $a_{\mathrm{HPF}, l}$ & -2.812 \\
Filter coefficient & $a_{\mathrm{HPF}, 2}$ & 2.641 \\
Filter coefficient & $a_{\mathrm{HPF}, 3}$ & -0.828 \\
Filter threshold [Nm] & $\tau_{\mathrm{HPF}, 0}$ & 40 \\
Momentum observer threshold [Nm] & $\tau_{\mathrm{MO}, 0}$ & 30 \\
Momentum observer low-pass cut-off [rad/s] & $K_{\mathrm{LP}}$ & 500 \\
Momentum observer high-pass cut-off [rad/s] & $K_{\mathrm{LP}}$ & 100 \\
Accelerometer threshold [g] & $\tau_{\mathrm{acc}, 0}$ & 1 \\
Load cell load arm [mm] & $L_{\mathrm{LC}}$ & 300 \\
Gravitational force [N] & $M_{g}$ & 40 \\
Center of gravity load arm [mm] & $L_{\mathrm{COG}}$ & 225 \\
Motor side inertia [kgm ${ }^{2}$ ] & $J_{m}$ & 2.5 \\
Load side inertia [kgm $\left.{ }^{2}\right]$ & $J_{l}$ & 0.2 \\
Clutch threshold torque [Nm] & $\tau_{\text {tr }}$ & 240 \\
Clutch residual torque [Nm] & $\tau_{\text {res }}$ & 60 \\
Calculated ratio [\%] & $\tau_{\text {res }} / \tau_{\text {tr }}$ & 25 \\
\hline
\end{tabular}

depending on the load position $q_{l}, \tau_{m}$ the motor torque and $\tau_{f}^{\prime}$ the friction torque of the motor and harmonic drive. Note that the flexibility of the joint due to the inherent compliance of a gearbox and/or clutch was neglected in order to reduce model complexity.

In nominal operation with an engaged overload clutch, the motor and load positions are equal, $q_{m}=q_{l}$. If the clutch torque $\tau_{c}$ is eliminated from Eq. (3) and all friction components are combined in a single friction term $\tau_{f}=\tau_{f}^{\prime}+D_{l} \dot{q}_{l}$, a single equation is obtained:

$$
\left(J_{l}+J_{m}\right) \ddot{q}_{l}+\tau_{f}+g\left(q_{l}\right)=\tau_{m}+\tau_{\mathrm{ext}}
$$

The bandpass momentum observer as introduced in [11] extends the standard low-pass filter observer by adding a highpass filter. The sensitivity towards intended contact is reduced making it an appropriate tool to detect unexpected collisions. Applied to a single joint, the bandpass momentum observer $r(t)$ reads

$$
\begin{aligned}
r(t) & =K_{\mathrm{LP}}[p(t)-p(0)] \\
& -K_{\mathrm{LP}} \int_{0}^{t}\left[\tau_{m, \text { meas }}-\tau_{f}-g\left(q_{l}\right)+\left(1+\frac{K_{\mathrm{HP}}}{K_{\mathrm{LP}}}\right) r\right] d t \\
& -K_{\mathrm{LP}} K_{\mathrm{HP}} \int_{0}^{t}\left[\int_{0}^{t} r d t\right] d t
\end{aligned}
$$

with $K_{\mathrm{LP}}$ and $K_{\mathrm{HP}}$ tuning parameters equal to the low-pass and high-pass filter (angular) cut-off frequencies respectively, $p(t)=\left(J_{l}+J_{m}\right) \dot{q}_{m \text {,meas }}$ the total momentum and $\tau_{m, \text { meas }}$ the measured motor torque. If $K_{\mathrm{LP}} \rightarrow \infty$ and $K_{\mathrm{HP}} \rightarrow 0$, then $r \rightarrow \tau_{\text {ext }}$. A $15 \mathrm{~Hz}$ high-pass filter cut-off frequency was chosen as in the data-driven detection methods. The low-pass filter frequency was set at $80 \mathrm{~Hz}$. A higher frequency did not

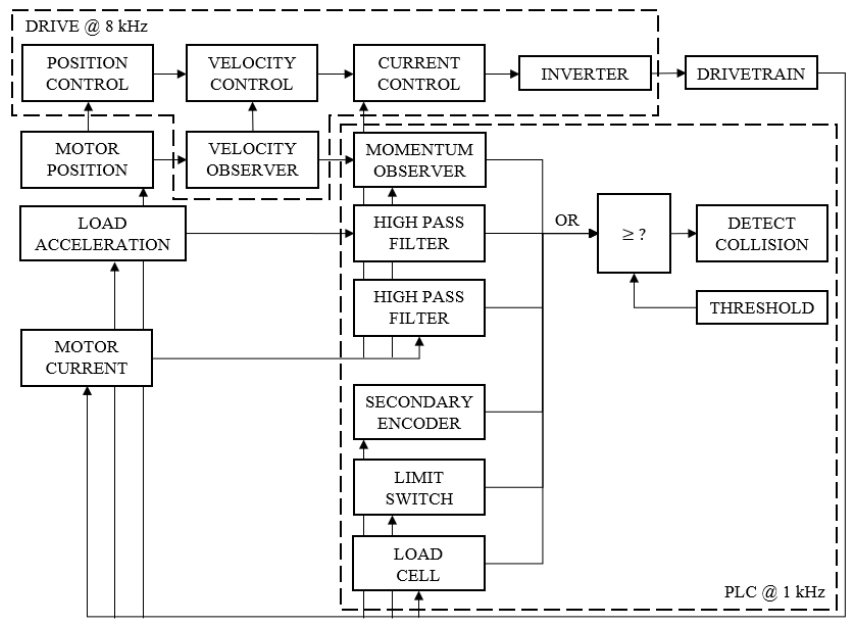

Fig. 1. Schematic overview of the control strategy, including the control loop and different collision detection techniques.

adequately reduce noise while a lower value introduced longer delays. Further details can be found in [11]. The momentum observer can be compared with a dynamic threshold $\tau_{\mathrm{MO}, 0}$ in a similar fashion as with the data-driven technique in order to detect collisions.

The velocity is obtained by differentiating position encoder data. The differentiation introduces noise in the momentum observer through $p(t)$. Low-pass filtering reduces this noise but introduces delay. In the experiments to follow, the same velocity estimate is used as input in the PID position control loop. The controller's D-term amplifies this noise. As such, an additional source of noise is introduced in the momentum observer via the motor torque $\tau_{m \text {,meas }}$. Reducing the D-action is no option however. High-speed robots are build for precision and speed which translates in aggressive tuning (while still ensuring system stability). In the experiments to follow, a velocity observer is used to reduce noise while avoiding delay. The differentiated position data is low-pass filtered with frequency $f_{\mathrm{VO}}=500 \mathrm{~Hz}$. In order to avoid the inherent delay, it is mended by adding a high-pass filtered modelbased estimate (with the same cut-off frequency $f_{\mathrm{VO}}$ ) of the velocity $\dot{q}_{l, \mathrm{HP}}=\int_{0}^{t} \tau_{m, \text { meas }} /\left(J_{l}+J_{m}\right) d t$ where a simplified version of Eq. (4) is used, including solely the inertia term. Kalman filtering could potentially be used as an alternative (similar as in [15]). The former method was chosen as it was already available as advanced option in the drive used for the experiments and runs at a higher sample frequency than a Kalman filter, which had to be implemented in the PLC control unit.

Note that the latter technique to estimate the velocity will also improve the data-driven collision detection based on motor currents, as discussed in Section II-C. A summary of the complete control and detection strategy is schematically represented in Fig. 1 . 


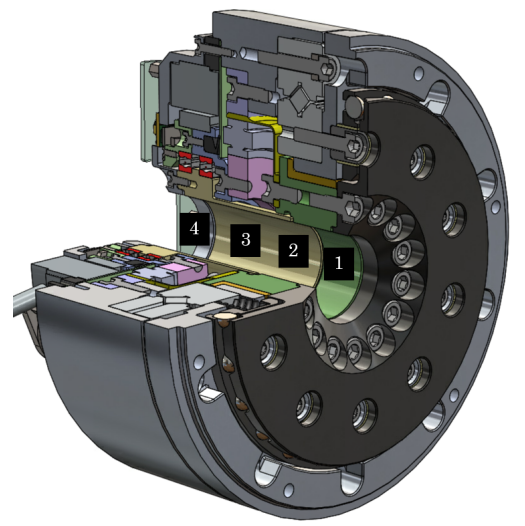

Fig. 2. Cross section of a high-speed industrial robot actuator with overload clutch. The actuator consists of a motor (4), a harmonic drive gearbox (3), a cross roller bearing (2) and the CFCC (1).

\section{EXPERIMENTAL COMPARISON OF COLLISION DETECTION TECHNIQUES}

Protecting the internal drive train of a high-speed industrial robot actuator equipped with a recently presented overload clutch [1] serves as use case for comparing collision detection techniques. A cross-section of the custom designed highspeed industrial robot actuator with CFCC that will be used as experimental platform for the evaluation of the collision detection techniques is shown in Fig. 2. It shows the series connection of (4) a motor (Tecnotion QTR-133-25) with absolute encoder (RLS AksIM-2, 20 bit magnetic encoder), (3) harmonic drive (CSD-40-50-2A) with reduction $N=50$ and overload clutch (1). The input and output side of the clutch (and by extension the actuator) can move with respect to each other thanks to the cross roller bearing (2). If excessive torque is applied on the output side of the clutch, the clutch disengages, partially decoupling the harmonic drive output (flex spline) and hence protecting it from overload. The actuator has an outer diameter of $174 \mathrm{~mm}$. Its peak torque output is $240 \mathrm{Nm}$ as this is the maximum torque the clutch can transmit without decoupling. Higher output speeds (300 $\% / s$ ) than a collaborative robot actuator can be achieved.

\section{A. Experimental setup}

The experimental setup shown in Fig. 3 consists of the actuator with CFCC on a test stand. A load arm is bolted to the output flange of the actuator. The load arm carries a $3.5 \mathrm{~kg}$ weight. Its tip compresses a TE Connectivity load cell, model FC2311-0000-0500-L which is amplified with a Texas Instruments INA826 precision instrumentation amplifier. The load cell allows to measure force (and load torque once multiplied with the length of the load arm $L_{\mathrm{LC}}$ ). The setup is instrumented with a M8 x 1 NPN inductive sensor, a Beckhoff EP3752 accelerometer and a RLS RE36 13 bit incremental encoder. The drive train is driven by a Beckhoff AX5106 servo drive and TwinCAT 3. The IO is processed via a Beckhoff EK1100 EtherCAT coupler [17]. A state machine controls

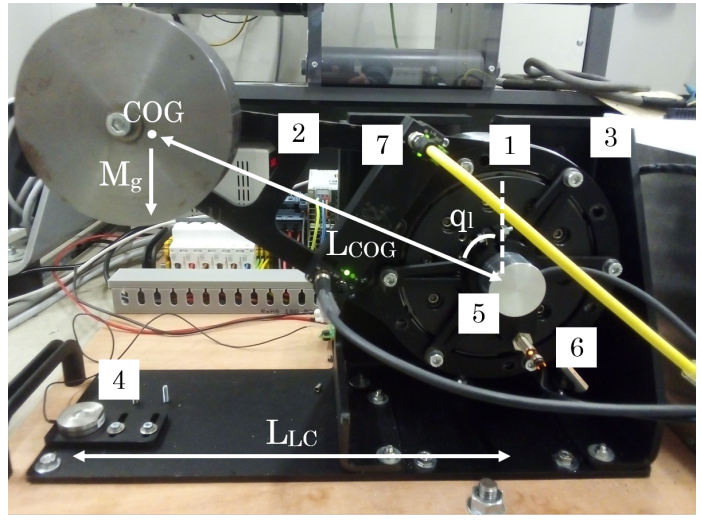

Fig. 3. Test setup showing the actuator with Combined Friction Cam Clutch (1) mounted on a test stand (3). A weight of $3.5 \mathrm{~kg}$ is attached to a load arm (2), on its turn bolted to the output flange of the actuator. The arm can swing until its tip touches a load cell (4). (5) is a secondary encoder, (6) a proximity sensor and (7) an accelerometer.

the setup and allows automatic execution of the collision experiments.

\section{B. Friction identification and gravity compensation}

A significant portion of the motor torque is converted to heat due to the friction torque $\tau_{f}$ in the bearings and harmonic drive gearbox. Friction was determined by measuring the motor torque while running at different constant speeds. The results are plotted in Fig. 4. A friction model including Coulomb and viscous terms

$$
\tau_{f}=D \dot{q}_{m}+\left\{\begin{array}{cc}
\min \left(\left|\tau_{m}\right|, \tau_{C}\right) \dot{q}_{m} / \epsilon & \text { if }\left|\dot{q}_{m}\right| \leqslant \epsilon \\
\operatorname{sign}\left(\dot{q}_{m}\right) \tau_{C} & \text { otherwise }
\end{array}\right.
$$

was fitted to the data resulting in $D=5 \mathrm{Nms} / \mathrm{rad}$ and $\tau_{C}=35$ $\mathrm{Nm}$ (reduced to the load side) with $\epsilon=0.03 \mathrm{rad} / \mathrm{s}$ introduced for computational purposes [18]. A higher order polynomial fits the data with smaller error. Its equation is added in the figure. The higher order polynomial fitted data was used.

Based on the dimensions defined in Fig. 3, the gravity torque $g\left(q_{l}\right)$ is given by

$$
g\left(q_{l}\right)=M_{g} L_{\mathrm{COG}} \sin \left(q_{l}\right)
$$

with $M_{g}$ the gravitational force and $L_{\mathrm{COG}}$ the load arm length to the Center Of Gravity (COG). Their values are specified in Table 【

\section{High speed collision experiment}

A high-speed $(300 \% / s)$ collision experiment is shown in Fig. 5 The following events occur:

1) Reversing from $0^{\circ} \rightarrow 80^{\circ} \rightarrow 0^{\circ}$ with a top speed of 300 $\% / s$ without collision. This reversing sequence is used to tune the collision detection thresholds in order to avoid false positives;

2) Moving from $0^{\circ} \rightarrow 110^{\circ}$ with a top speed of $300^{\circ} / \mathrm{s}$. The tip of the load arm collides with the load cell.

Fig. 5 shows the actual motor position, speed and acceleration as reduced to the load side and the different measurements 


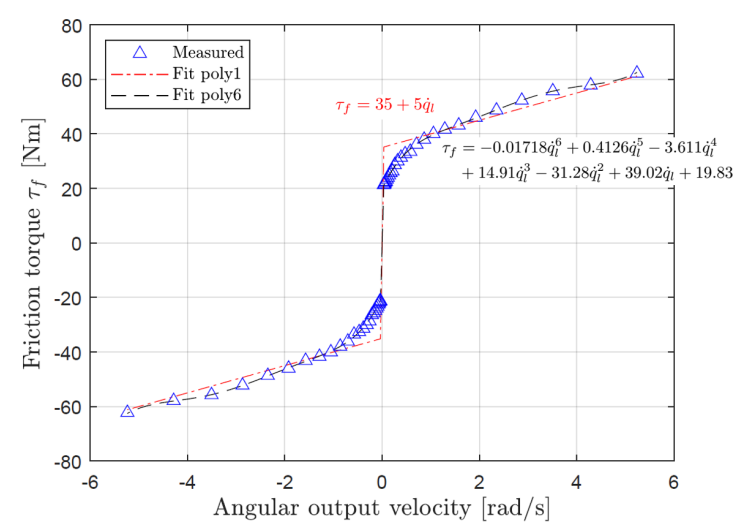

Fig. 4. Identification of the friction torque. A first order ('poly1') and sixth order polynomial ('poly6') are fitted to the data.
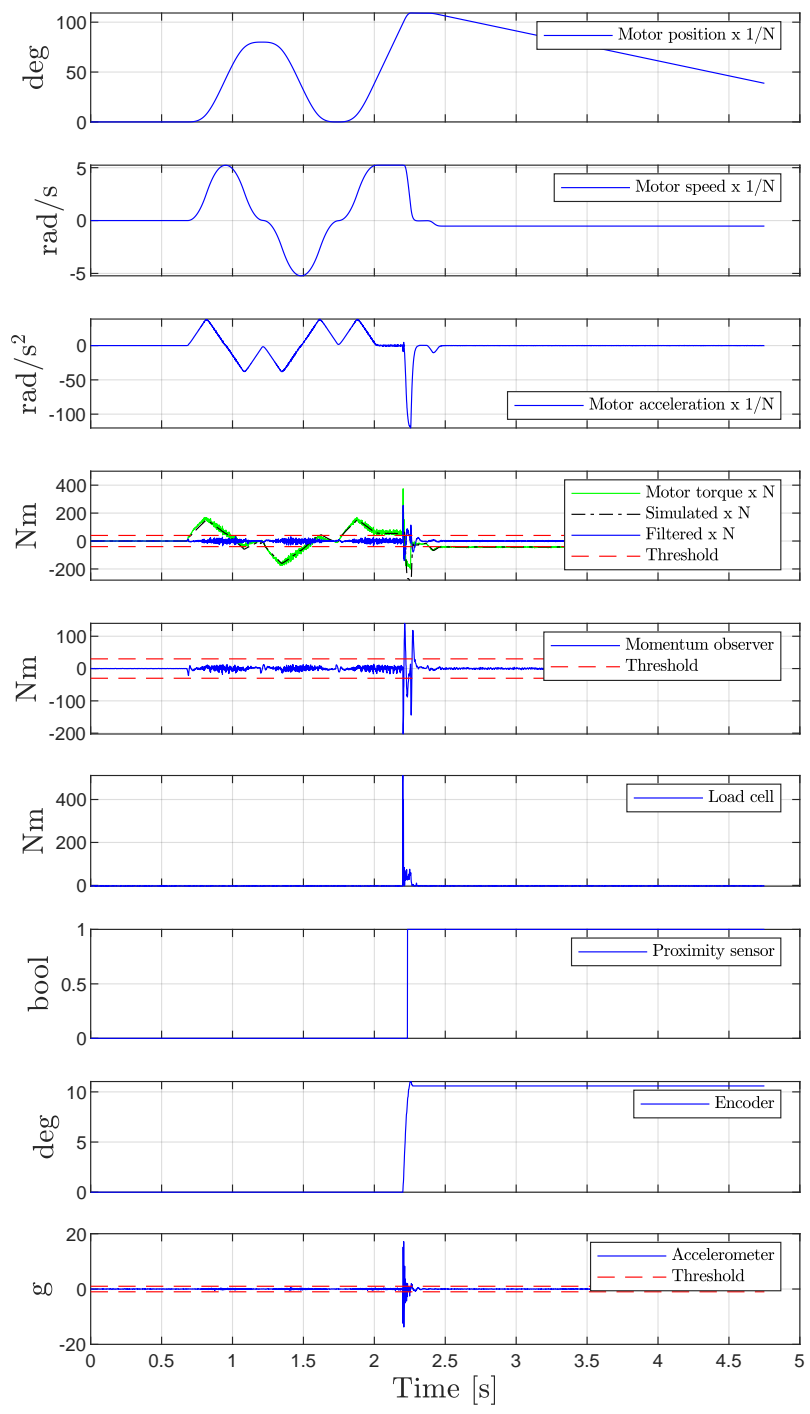

Fig. 5. High speed collision test. The actuator collides with the load cell at high speed $\left(300^{\circ} / \mathrm{s}\right)$. The latter occurs at $2.2 \mathrm{~s}$. The CFCC disengages and limits the torque transmitted from the load to the motor side of the actuator. The collision is detected and the motor inertia is braked. used to detect a collision. These are the high-pass-filtered motor torque, the momentum observer, the load cell output, the proximity switch output, the relative angular displacement as monitored by the secondary encoder and the accelerometer data. The torque as detected by the load cell is close to 500 $\mathrm{Nm}$. Depending on the load inertia to be decelerated, this torque can be above the specifications of the harmonic drive (the HD CSD-40-50-2A gearbox has a maximum repeatable output torque of $281 \mathrm{Nm}$ and a maximum allowed emergency stop torque of $480 \mathrm{Nm} \mathrm{[19]}$ ) and could result in mechanical failure of its flex spline. Due to the presence of the overload clutch, such a scenario is avoided. Those excessive collision loads result in decoupling of the overload clutch. The maximum collision torque as 'experienced' by the harmonic drive and motor is the clutch threshold torque.

All techniques allow to detect the collision. None of them falsely identifies a collision in the reversing sequence $0^{\circ} \rightarrow$ $80^{\circ} \rightarrow 0^{\circ}$. The same peak torque was measured by the load cell for all techniques. Hence not the detection techniques but the kinetic energy upon collision and the deceleration time of the motor inertia determine the measured external torque. The same holds for the final relative angular displacement of the clutch input and output flange. It was measured to be approximately $11^{\circ}$ independent of the collision detection technique. The detection techniques differ significantly in other metrics such as the threshold torque and sensitivity. These differences are discussed next.

\section{Comparison of collision detection techniques}

Table II summarizes several important metrics for the different collision detection techniques. The unit used to list the threshold depends on the measurement: the load cell, highpass-filter and momentum observer thresholds are expressed in $\mathrm{Nm}$, the proximity switch and encoder in degrees and the accelerometer in g's. The ratio of peak by threshold value is a unitless and quantitative metric to rank the sensitivity of the collision detection techniques. As all data was gathered in the same experiment, the techniques that measured values significantly larger than the threshold are the most sensitive. Stated otherwise: the higher the sensitivity, the lower the minimal kinetic energy a collision can have and still be detected. The delay of the detection is measured with respect to the technique with the highest sensitivity.

1) Load cell: Detection based on the external load cell data is the most accurate and direct way of detecting a collision. This method serves as benchmark for the other techniques. A threshold as low as $2 \mathrm{Nm}$ can be chosen. This is well below the clutch threshold torque $\tau_{\text {tr }}=240 \mathrm{Nm}$. The peak/threshold ratio is 250 .

2) Sensorless/proprioceptive techniques: The clutch threshold torque $\tau_{\text {tr }}$ has to be larger than the dynamic collision detection threshold as it is the maximum torque transferred to the motor side in the event of a collision. The maximum transferred torque must exceed the collision detection threshold otherwise the motor is not aware of the collision and does not react appropriately. In order to avoid false positives, 
TABLE II

COMPARISON OF DIFFERENT COLLISION DETECTION TECHNIQUES.

\begin{tabular}{|c|c|c|c|c|c|c|}
\hline Metric & $\begin{array}{l}\overline{\bar{J}} \\
\bar{\Xi} \\
\stackrel{0}{\Xi}\end{array}$ & 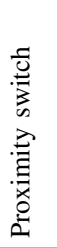 & $\begin{array}{l}\overline{\bar{v}} \\
\overline{0} \\
\tilde{U}\end{array}$ & 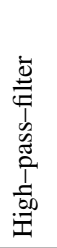 & 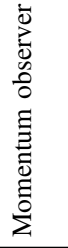 & 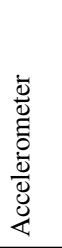 \\
\hline Threshold [Nm] & 2 & - & - & 40 & 30 & - \\
\hline Threshold $\left[{ }^{\circ}\right]$ & - & 0.5 & 0.1 & - & - & - \\
\hline Threshold high speed $[\mathrm{g}]$ & - & - & - & - & - & 1.0 \\
\hline Peak/threshold & 250 & 22 & 110 & 6.3 & 6.7 & 16.0 \\
\hline Delay [ms] & 0 & 10 & 2 & 2 & 2 & $\leqslant 1$ \\
\hline Detect if not disengaged & yes & no & no & yes & yes & yes \\
\hline Cost* & +++ & + & ++ & 0 & 0 & ++ \\
\hline Computation* & + & + & + & ++ & +++ & ++ \\
\hline
\end{tabular}

*The more ' + ', the higher the cost/computation time.

the threshold for the high-pass-filter technique was set to 40 $\mathrm{Nm}$. A delay of $2 \mathrm{~ms}$ with respect to the load cell detection technique was measured.

The thresholds used for the momentum observer depend on the cut-off frequencies $K_{\mathrm{LP}}$ and $K_{\mathrm{HP}}$. A lower $K_{\mathrm{LP}}$ filters the high-frequent component in the motor torque introduced by the motor controller but introduces additional detection delay. Using $K_{\mathrm{LP}}=80 \mathrm{~Hz}$ and $K_{\mathrm{HP}}=15 \mathrm{~Hz}$, a sensitivity of 6.7 was found, which is similar to the high-pass-filter technique. A delay of $2 \mathrm{~ms}$ was observed.

3) Proximity switch and secondary encoder: The proximity switch and the secondary encoder measure the angular displacement of the input and output side of the clutch. Such a displacement occurs only upon disengagement of the clutch. Collisions that do not result in disengagement are not detected. The external torque must therefore be larger than the threshold torque of the clutch, $\tau_{\text {tr }}=240 \mathrm{Nm}$ in this case. Only the highspeed collision resulted in clutch disengagement. Although insensitive to low-speed collisions, they have the highest sensitivity once the clutch decouples. The detection with the secondary encoder and the proximity switch showed a delay of 2 and $10 \mathrm{~ms}$ respectively. The switch detects whether there is material in front of it or not. Positioning the switch at the edge material/no material and hence detection/no detection is nearly impossible to do with the same accuracy of even a low resolution encoder.

4) Accelerometer: The accelerometer proved to be the most sensitive collision detection technique without decoupling of the clutch. The detection threshold was $1.0 \mathrm{~g}$ and sensitivity 16.0. No collision detection delay was measured.

\section{CONCLUSION}

Different collision detection techniques originally developed in the context of safe human-robot collaboration were evaluated at higher speeds for the application of high-speed industrial robots. Protecting the internal drive train of a highspeed industrial robot actuator equipped with an overload clutch served as use case in this study. Collision detection using proximity switches or a secondary encoder require the clutch to disengage prior to detection. Collision detection based on high-pass filtering of motor currents and by applying the bandpass momentum observer showed similar collision sensitivity. Collision detection based on accelerometer data has the highest collision detection sensitivity if the clutch does not disengage upon impact.

\section{REFERENCES}

[1] F. Ostyn, T. Lefebvre, B. Vanderborght, and G. Crevecoeur. Overload clutch design for collision tolerant high-speed industrial robots. IEEE Robotics and Automation Letters, 6(2):863-870, 42021.

[2] S. Haddadin, A. Luca, and A. Albu-Schaffer. Robot collisions: A survey on detection, isolation, and identification. IEEE Transactions on Robotics, PP:1-21, 102017.

[3] D. Seok, Y. B. Kim, S. Y. Lee, J. Kim, and H. R. Choi. Ultra-thin joint torque sensor with enhanced sensitivity for robotic application. IEEE Robotics and Automation Letters, 5(4):5873-5880, 2020.

[4] P. Wisanuvej, J. Liu, C. Chen, and G. Yang. Blind collision detection and obstacle characterisation using a compliant robotic arm. In 2014 IEEE International Conference on Robotics and Automation (ICRA), pages 2249-2254, 2014.

[5] L. Richter and R. Bruder. Design, implementation and evaluation of an independent real-time safety layer for medical robotic systems using a force-torque-acceleration (fta) sensor. International Journal of Computer Assisted Radiology and Surgery, 8:429-436, 2012.

[6] M. Geravand, F. Flacco, and A. De Luca. Human-robot physical interaction and collaboration using an industrial robot with a closed control architecture. pages 4000-4007, 052013.

[7] M. Indri, S. Trapani, and I. Lazzero. Development of a virtual collision sensor for industrial robots. Sensors, 17:1148, 052017.

[8] P. Koustoumpardis A. Sharkawy and N. Aspragathos. Neural network design for manipulator collision detection based only on the joint position sensors. Robotica, pages 1-19, 062019.

[9] S. Mamedov and S. Mikhel. Practical aspects of model-based collision detection. Frontiers in Robotics and AI, 7:162, 112020.

[10] A. De Luca, A. Albu-Schaffer, S. Haddadin, and G. Hirzinger. Collision detection and safe reaction with the dlr-iii lightweight manipulator arm. In 2006 IEEE/RSJ International Conference on Intelligent Robots and Systems, pages 1623-1630, 2006.

[11] J. Kim C. Cho, J. Song Y. Kim, and J. Kyung. Collision detection algorithm to distinguish between intended contact and unexpected collision. Advanced Robotics, 26:1-16, 112012.

[12] S. Lee and J. Song. Sensorless collision detection based on friction model for a robot manipulator. International Journal of Precision Engineering and Manufacturing, 17:11-17, 012016.

[13] J. Ye Z. Li and $\mathrm{H}$. Wu. A virtual sensor for collision detection and distinction with conventional industrial robots. Sensors, 19(10):2368, 2019.

[14] J. Jankowski G. Garofalo, N. Mansfeld and C. Ott. Sliding mode momentum observers for estimation of external torques and joint acceleration. In 2019 International Conference on Robotics and Automation (ICRA), pages 6117-6123, 2019.

[15] J. Kühn S. Birjandi and S. Haddadin. Observer-extended direct method for collision monitoring in robot manipulators using proprioception and imu sensing. IEEE Robotics and Automation Letters, 5(2):954-961, 2020.

[16] S. Birjandi and S. Haddadin. Model-adaptive high-speed collision detection for serial-chain robot manipulators. IEEE Robotics and Automation Letters, 5(4):6544-6551, 2020.

[17] K. Langlois, T. van der Hoeven, D. Rodriguez Cianca, T. Verstraten, T. Bacek, B. Convens, C. Rodriguez-Guerrero, V. Grosu, D. Lefeber, and B. Vanderborght. Ethercat tutorial: An introduction for realtime hardware communication on windows [tutorial]. IEEE Robotics Automation Magazine, 25(1):22-122, 2018.

[18] A. Albu-Schäffer. Regelung von Robotern mit elastischen Gelenken am Beispiel der DLR-Leichtbauarme. $\mathrm{PhD}$ thesis, Technischen Universität München, 2001.

[19] Harmonic drive. https://harmonicdrive.de Accessed: 17.11.2020. 